\title{
Quiste dentígero: diagnóstico y resolución de un caso. Revisión de la literatura
}

\section{Dentigerous cyst: diagnosis and resolution of a case. A review of the literature}

\author{
Vázquez Diego J*, Gandini Pablo C**, Carvajal Eduardo E***
}

\begin{abstract}
RESUMEN
Se presenta un caso clínico de quiste dentígero asociado a un segundo premolar inferior derecho retenido. El paciente de 13 años de edad es de sexo masculino. Se utilizan las técnicas radiográficas extraoral lateral oblicua de rama y la intraoral del paralelo para poder localizar y diagnosticar presuntivamente dicha patología. Posteriormente se realiza la intervención quirúrgica para realizar la extracción del diente retenido y la enucleación del quiste el que es enviado para realizar los estudios anatomopatológicos los que corroboran el diagnóstico presuntivo. Se decide hacer seguimiento del caso. Basado en lo expuesto se analiza al quiste dentígero según ubicación, sexo, edad y maxilar; habiendo realizado una revisión de la literatura.
\end{abstract}

Palabras clave: Quiste dentígero, técnicas radiográficas, tratamiento quirúrgico, diente retenido, caso clínico.

\section{SUMMARY}

A clinical case of dentigerous cyst associated with an impacted lower right second premolar in a 13-year-old male patient, is reported. An extraoral oblique lateral technique of the ramus and a parallel intraoral technique were used to locate the cyst and to perform a presumptive diagnosis of the lesion. A surgical procedure was then carried out to remove the impacted tooth and also to enucleate the cyst, which was sent to the laboratory for a histo-pathological study; this study confirmed our earlier presumptive diagnosis. Finally, we decided to follow-up the case. Based on our findings, dentigerous cyst is analyzed according to its location, sex and age of patients and most affected jaw, after reviewing the literature on this subject.

Key words: Dentigerous cyst, radiographic techniques, surgical treatment, impacted tooth, clinical case.

Fecha de recepción: 14 de mayo de 2008.

Aceptado para publicación: 22 de mayo de 2008.

* Jefe de Trabajos Prácticos Regular. Cátedra de Radiología. Facultad de Odontología. Universidad de Buenos Aires. Argentina. Especialista en Diagnóstico por Imágenes Buco-máxilo-facial.

* Ayudante de Primera Regular. Cátedra de Cirugía B. M. F. III. Facultad de Odontología-Universidad de Buenos Aires. Argentina. Especialista en Cirugía Buco-máxilo-facial.

*** Profesor Adjunto Regular. Cátedra de Radiología. Facultad de Odontología. Universidad de Buenos Aires. Argentina. Especialista en Diagnóstico por Imágenes Buco-máxilo-facial.

Vázquez Diego J, Gandini Pablo C, Carvajal Eduardo E. Quiste dentígero: diagnóstico y resolución de un caso. Revisión de la literatura. Av. Odontoestomatol 2008; 24 (6): 359-364.

\section{INTRODUCCIÓN}

Los quistes son cavidades patológicas de contenido líquido, semilíquido, sólido o gaseoso, revestidas de epitelio en su cara interior y por tejido conectivo en el exterior. Los de origen embrionario derivan de los restos de Malassez que son restos de la vaina epitelial radicular de Hertwig que persisten en el ligamen- 
to periodontal después de completarse la formación de la raíz, el epitelio del órgano del esmalte que es un epitelio residual que rodea la corona del diente después de completarse la formación del esmalte y de restos de la lámina dental que son islotes y tiras de epitelio que se originan en el epitelio oral y permanecen en los tejidos después de inducir el desarrollo del diente. $(1,2,3,9)$

El quiste dentígero o coronodentario es clasificado por la O.M.S. en 1992 como uno de los quistes odontogénicos de desarrollo, siendo una cavidad anormal que rodea la corona de un diente no erupcionado, dejando por fuera la raíz o raíces de dicha estructura dentaria. Se origina por alteración del epitelio del órgano del esmalte después de la formación completa de la corona por la acumulación de líquido entre las capas del epitelio adamantino o entre este y la corona dental. Está siempre asociado a la corona de un diente retenido. Suelen descubrirse por un estudio radiográfico de rutina. Es de crecimiento lento y asintomático. Pueden originar expansión del hueso, asimetría facial, gran desplazamiento de los dientes y gran reabsorción radicular de los dientes adyacentes. $(3,9,14,18)$

Suelen ocurrir en el maxilar inferior en mayor proporción respecto al maxilar superior. Las piezas mas afectadas suelen ser los terceros molares y los caninos en ambos maxilares. En el maxilar inferior a consecuencia de este quiste, en la zona molar puede llegar a producirse una resorción casi total de la rama ascendente, con el desplazamiento del molar. En el maxilar superior en la zona del canino produce expansión de la porción anterior de dicho maxilar y puede semejar una celulitis o a una sinusitis, aunque puede producir una sinusitis por invasión del antro sinusal. Puede también progresar hacia las fosas nasales y a la fosa pterigomaxilar produciendo dolor e infección y en ocasiones supuración y fístulas al exterior.

Suele haber mayor predominio en el sexo femenino sobre el masculino y una mayor incidencia de los 20 a 40 años de edad. $(4,5,6,7,11)$

Radiográficamente se observa una zona radiolúcida redondeada y bien circunscrita asociada a la corona del diente sin erupcionar. El tratamiento es la enucleación quirúrgica y existe la posibilidad de recu- rrencia si dicha remoción es incompleta dado su comportamiento quístico.

El diagnóstico diferencial se realiza con ameloblastoma, carcinoma epidermoide o el carcinoma mucoepidermoide. $(8,10,12,13,14)$

El objetivo de este trabajo es la presentación de un caso clínico de quiste dentígero asociado a un segundo premolar inferior retenido través del diagnóstico radiográfico y su tratamiento quirúrgico y una revisión de la literatura científica.

\section{MATERIALES Y MÉTODOS}

Se realizó el estudio radiográfico extraoral con un equipo marca Trophi con valores utilizados de $70 \mathrm{Kv}$, $8 \mathrm{~mA}$, tiempo de exposición: 0,8 segundos.

La película utilizada para esta técnica es de $13 \mathrm{~cm}$ por $18 \mathrm{~cm}$ marca Agfa y chasis rígido con pantallas reforzadoras sensible al espectro de luz verde.

El estudio radiográfico intraoral se realizó con un equipo radiográfico marca Dabi Atlante con valores fijos de $70 \mathrm{Kv}, 8 \mathrm{~mA}$ y tiempo de exposición de 0,5 seg. Se utilizaron películas retroalveolares de $3 \mathrm{~cm}$ por $4 \mathrm{~cm}$ marca Kodak. Para el procesado se utilizó una máquina automática marca AT/2000.

Se utilizaron los elementos de protección según normas vigentes.

El tratamiento quirúrgico se llevó a cabo con material de antisepsia descartable, y para la confección de los campos. Anestesia local: 4 anestubos marca totalcaína, aguja de anestesia corta mango de bisturí $\mathrm{N}^{\circ} 3$, hoja $\mathrm{N}^{\mathrm{O}} 15$; separadores de farabeuf, periostótomo, sindesmótomo, fresas quirúrgicas redondas números: 4, 6 y 8, instrumental rotatorio con su fisiodispenser, pinzas Pean, Kocher, gubia. Cucharas y limas para hueso, hilo de sutura 0000, porta aguja, tijera, gasa estéril, solución fisiológica y solución desinfectante.

Los estudios histopatológicos se realizaron en el Servicio de Anatomía Patológica del San Roque de Gonnet dependiente del Ministerio de Salud de la Provincia de Buenos Aires. 


\section{CASO CLÍNICO}

Concurre a la consulta un paciente de sexo masculino de 13 años, derivado del ortodoncista, ya que al realizar la radiografía de rutina encuentra una pieza dentaria retenida y a su alrededor una imagen radiolúcida rodeando al diente retenido, para continuar su tratamiento deriva al paciente para realizar su extracción (Fig. 1).

Se realiza la toma de una técnica lateral oblicua de rama derecha, que es una técnica radiográfica extraoral que nos permitirá ver con amplitud la zona en cuestión y sus estructuras aledañas, en la que se observa la persistencia del segundo molar temporario en boca, la pieza 4.5 retenida y una zona radiolúcida circunscripta rodeando a la pieza retenida (Fig. 1).

A posterior se decide realizar una técnica de cilindro largo, radiografía intraoral que nos permitirá ver con

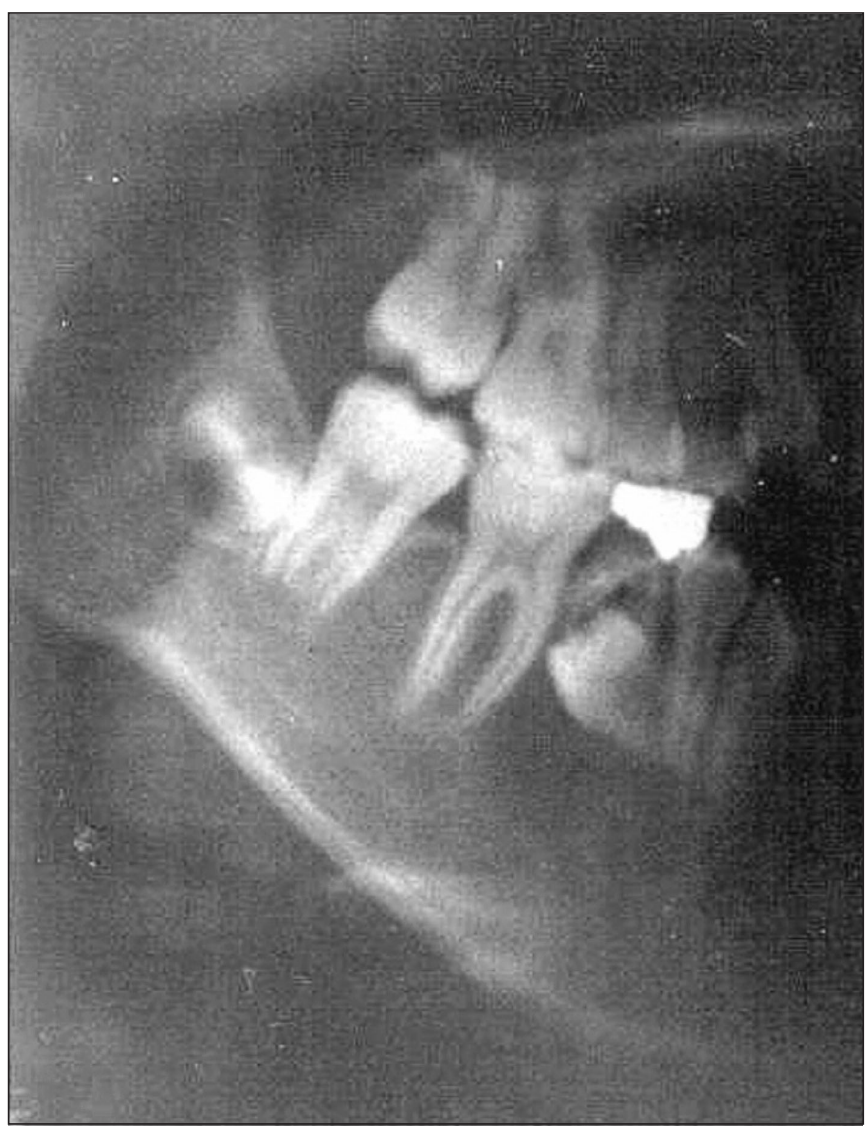

Fig. 1. mayor isometría e isomorfismo la lesión que rodea al diente con la cortical radiopaca de límites netos la que podríamos diagnosticar presuntivamente como quiste dentígero (Fig. 2).

Realizados todos lo estudios de rutina se decide su remoción quirúrgica. Se presentan las fotos de lo distintos pasos.

En la figura 3 se observa el molar inferior temporario que persiste todavía en la cavidad bucal, se realiza un colgajo mucoperióstico para abordar el supuesto quiste que rodea a la corona de la pieza 4.5 retenida.

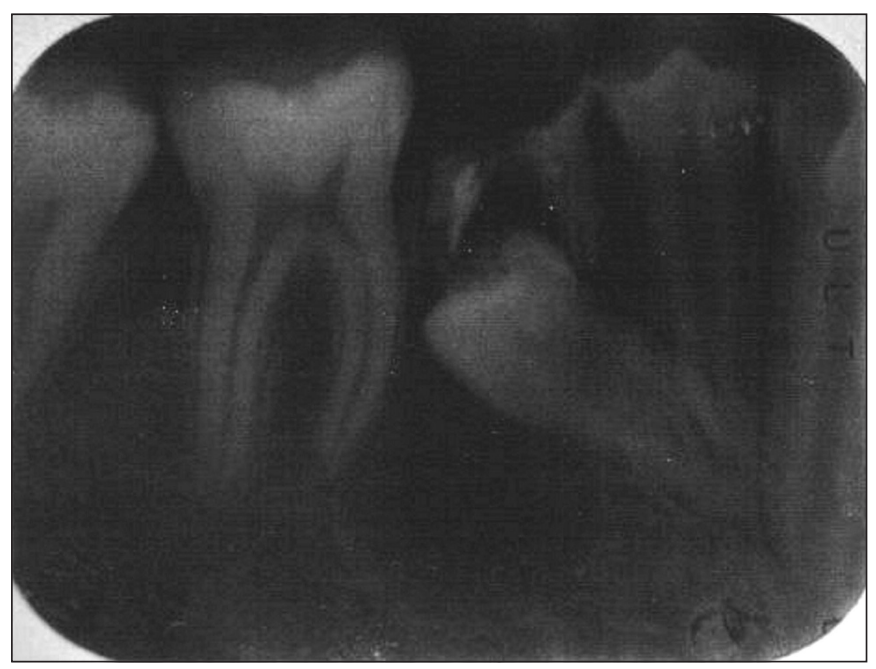

Fig. 2.

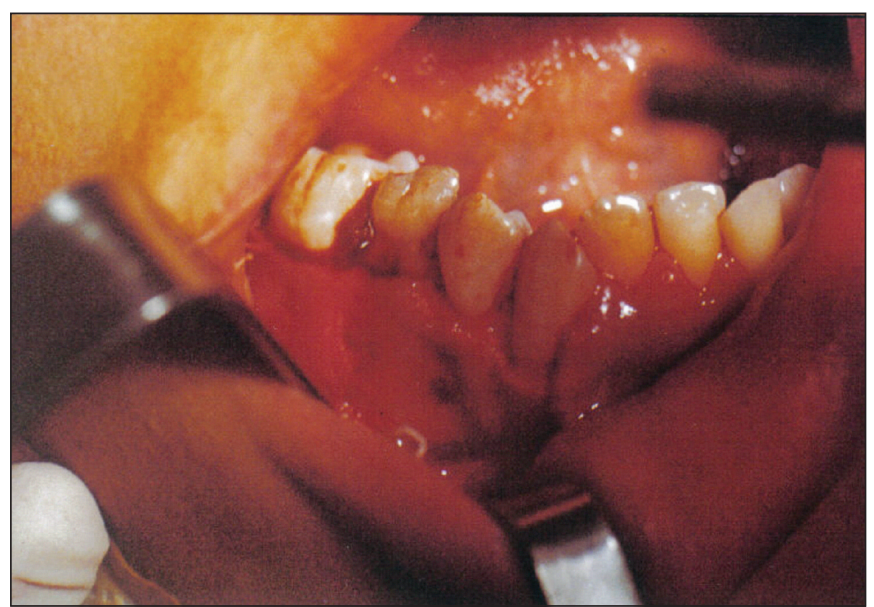

Fig. 3. 
En la figura 4 se observa una vez realizada la extracción del 8.5 , se realiza una pequeña osteotomía para permitir la exodoncia de la pieza 4.5.

En la figura 5 se realiza el enucleado de la membrana quística que es de gran consistencia, en la figura 6 se observa la reposición del colgajo y la sutura post operatoria. La figura 7 nos muestra el segundo molar temporario, el segundo premolar permanente y el contenido quístico una vez extraído.

Se realiza el estudio anatomopatológico de la pieza quirúrgica.

El informe anatomopatológico diagnostica en la muestra un sector tisular con revestimiento de epite-

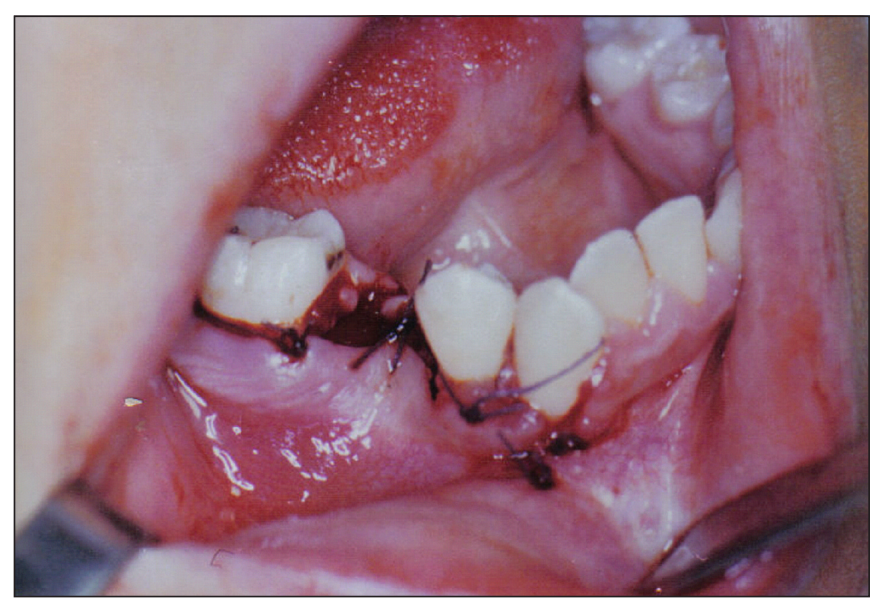

Fig. 6.

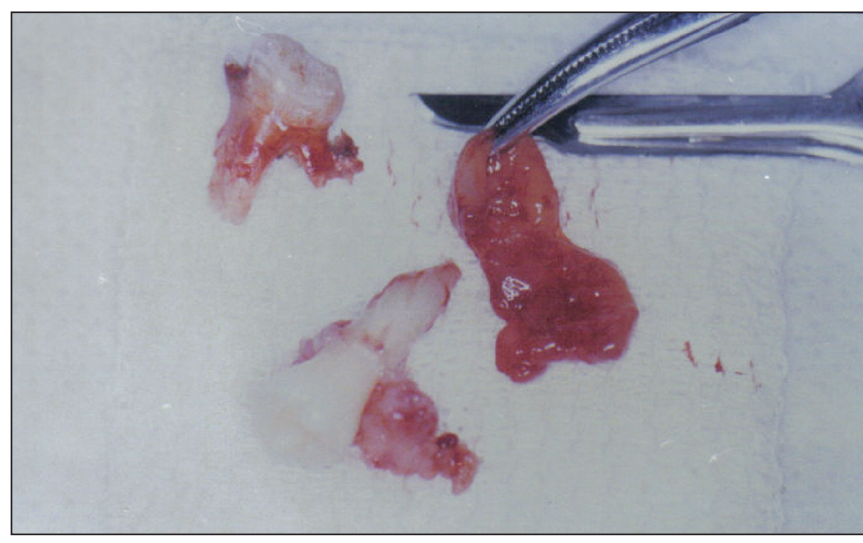

Fig. 7.

Fig. 4.

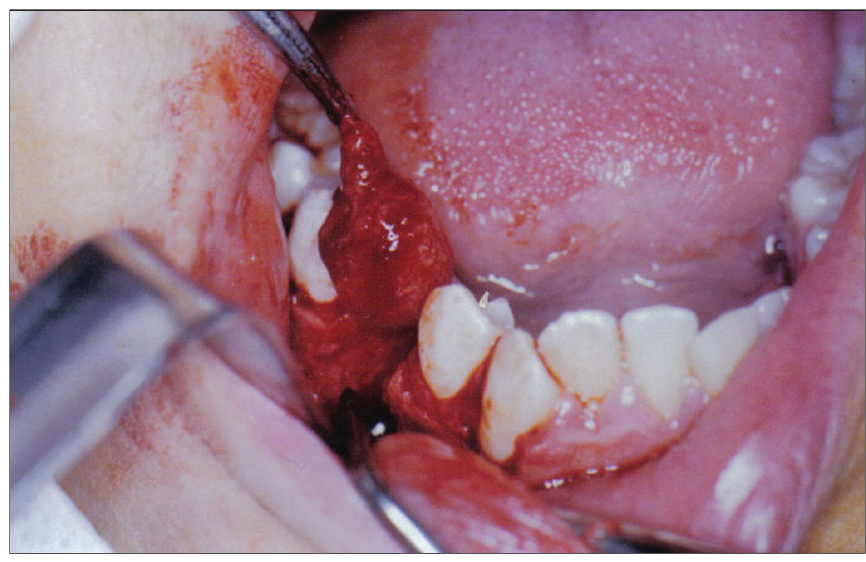

Fig. 5. lio escamoso estratificado delgado, con producción de esferas acidófilas focales. El corion muestra edema e infiltrados linfoplasmocitarios reactivos y en la periferia presenta fibrosis y lamina de hueso reactivo concluyendo con el diagnóstico de quiste dentígero.

\section{DISCUSIÓN Y CONCLUSIÓN}

Se debe destacar la importancia de un diagnóstico temprano de esta afección debido a la posibilidad de poder originar expansión del hueso, asimetría facial, gran desplazamiento de los dientes y gran reabsorción radicular de las piezas adyacentes. La posibilidad de una radiografía extraoral como puede ser una lateral de rama en el caso de no poseer un equipo 
panorámico nos permite observar ambos maxilares y zonas aledañas a ellas, como en este caso en el que se visualiza el diente retenido y el quiste rodeándolo. Es una técnica sencilla, poco costosa y fácil de realizar. La técnica intraoral del paralelo o cilindro largo nos permite evitar superposiciones y defectos de tamaño en la que se visualiza una cavidad radiolúcida con cortical de límites netos, rodeando la corona del diente retenido.

Los quistes dentígeros suelen ser, después de los apicales y residuales, los de mayor incidencia entre los quistes odontogénicos, nuestro caso clínico es de un paciente de 13 años y de sexo masculino lo que no suele coincidir con las estadísticas encontradas en la literatura (mayor incidencia de 20 a 40 años y el sexo femenino mas frecuente que el masculino), (11) pero coincide en el maxilar siendo el inferior en el que se han diagnosticado más apariciones de este quiste, respecto del superior. $(11,19$, 21, 22)

Por lo expuesto, hemos desarrollado un caso clínico con una pieza dentaria retenida y un quiste dentígero en relación a ella y hemos descrito algunas de la técnicas radiográficas útiles para su diagnóstico, destacando la técnica intrabucal del paralelo o de Fitzgerald por su isomorfismo e isometría y a la técnica lateral oblicua de rama que nos permite obtener una visión general la que relaciona la lesión en estudio con el resto de las arcada dentaria, como así también descartar otras patologías en relación con el estudio en cuestión. También es de destacar una correcta utilización de las normas de bioseguridad en el la utilización de los rayos x y en el tratamiento quirúrgico y el cumplimiento de todos los pasos operatorios previamente planeados anteriores a la cirugía. La corroboración posterior del diagnóstico presuntivo a través de los estudios anatomopatológicos es imprescindible y los seguimientos a corto, mediano y largo plazo de dicho paciente son recomendados.

\section{BIBLIOGRAFÍA}

1. Regezi \& Sciubba: Quistes de la boca,: Patología Bucal 3era. ed. México, McGraw-Hill Interamericana, 1999.
2. Regezi \& Sciubba: Cysts of the Oral Region, in Regezi y Sciubba: Oral Pathology 2 nd ed. Philadelphia, PA, Saunders, 1993.

3. Sapp JPh, Eversole LR and Wysocki GP: Cysts of the oral region, in Sapp Eversole Wysocki: Contemporary Oral and Maxillofacial Pathology, St. Louis, Mosby, 1997.

4. Martínez Pérez D y Varela-Morales M: Conservative treatment of dentigerous cyst in children: a report of 4 cases. J Oral Maxillofac Surg 2001;59: 331-4.

5. Clauser C, Zuccati G, Barone R, et al: Simplified surgical orthodontic treatment of a dentigerous cyst. J Clin Orthod 1994;28:103.

6. Gardner DG. Plexiform unicystic ameloblastoma a diagnostic problem in dentigerous cysts. Cancer 1981;47:1358.

7. Johnson L, Sapp JP. Melntire DN. Squamous cell carcinoma arising in dentigerous cyst. J oral Maxillofac Surg, 1994;52:987-90.

8. Maximiw WG. Wood RE. Carcinoma arising in a dentigerous cyst: a case report and review of the literature. J. Oral Maxillofac Surg. 1991;49: 639-43.

9. Sapp JP. Eversole LR. Wysocki GP.. Patología Oral y Maxilofacial Contemporánea. 1999;20:23.

10. Joseph A. Regezi, D.D.S., M.S. Odontogenic Cysts, Odontogenic Tumors, Fibroosseous, and Giant Cell Lesions of the Jaws. Modern Pathology 2002;15:331-41.

11. Rancaño Medina Vega Comportamiento Epidemiológico de los quistes maxilares. Estudio de 10 años. 2003 May;95(5):632-5.

12. Concha, Guillermo. Radiología de quistes de los maxilares. Rev. Hosp. Clin. Univ. Chile 2002;13 (1):23-32.

13. Tami Moura, Lopez, Moustarih, Moretta, Montilla, Rivera. Quiste Dentigero. Revisión bibliográ- 
fica y presentación de un caso. Acta odontol Venez 2000; June 38(2).

14. Monserrat E. Quiste dentígero : presentación de un caso. Acta Odontol Venez 2002;40(2).

15. Usture E, Fitoz S, Atazoy C, Erden I, Aykar S. Bilateral maxillary dentigerous cyst: A case report. Oral Surg Oral Med Oral Pat hol Oral Radiol Endod 2002; 95(5):632-35.

16. Shah N, Thuan H, Beale I. Spontaneus regression of bilateral dentigerous cyst associated with impacted mandibular third molar. $\mathrm{Br} \mathrm{J}$ Dental 2002;26(192):75-6.

17. Tskumoto G, Sasaki K, Akiyama T. A radiologic analasys of dentigerous cyst and odontogénico keratocyst. Oral Surg Oral Med Oral Pathol Oral Radiol Endod 2001;91:743-7.

18. Olson J W, Miller R, Krushner G, Vest TM. Odontogenic carcinoma occurring in dentigerous cyst: A case report and clinical management. J Periodontal 2000;71:1365-70.

19. Quintana Díaz, Abdulsalam, López Lazo, Vega Basulto, Pinilla González y Hussein Quiste dentígeno gigante en una niña. Presentación de un caso. Rev Cubana Estomatol 2007; abr-jun 44(2) Ciudad de La Habana.

20. Allais de Maurette. Maurette O'Brien. Haiter-Neto Moraes. Tratamiento de quiste dentígero bilateral mandibular por medio de dos tipos de tratamientos. Relato de caso clínico y comparación entre la técnicas. Acta odontol Venez 2007;45

(1) Caracas ene.

21. Sands T, Tocchio C. Multiple dentigerous cysts in a child. Oral Health 1998;88:27-9.

22. Ko KS, Dover DG, Jordan RC. Bilateral dentigerous cysts-report of an unusual case and review of the literature. J Can Dent Assoc 1999; 65:49-51.

\section{CORRESPONDENCIA}

J. Vázquez Diego

Gdor. Marcelino Ugarte 1548, $1^{\circ}$ piso, departamento "C" Olivos.

C P 1636

Provincia de Buenos Aires. Argentina

E- Mail: jv983@hotmail.com.ar

eecarbajal@yahoo.com.ar 